\title{
THE ZEROS OF QUASI-ANALYTIC FUNCTIONS ${ }^{1}$
}

\author{
ARTHUR O. GARDER, JR.
}

\section{Statement of the theorem.}

Definition 1. $C\left(M_{n}, k\right)$ is the class of functions $f(x)$ for which there exist positive constants $A$ and $k$ and a sequence $\left(M_{n}\right)_{n=0}^{\infty}$ such that

$$
\left|f^{(n)}(x)\right| \leqq A k^{n} M_{n}, \quad-\infty<x<\infty, n=0,1,2, \cdots .
$$

Definition 2. $C\left(M_{n}, k\right)$ is a quasi-analytic class if 0 is the only element $f$ of $C\left(M_{n}, k\right)$ for which there exists a point $x_{0}$ at which $f^{(n)}\left(x_{0}\right)=0$ for $n=0,1,2, \cdots$.

We introduce the functions

$$
T(u)=\max _{n \geqq 0} u^{n}\left(M_{n}\right)^{-1}, \quad 0 \leqq u<\infty,
$$

and

$$
H(v)=\frac{2}{\pi} \int_{1}^{v} u^{-2} \log T(u) d u .
$$

The following property of $H(v)$ characterizes quasi-analytic classes.

TheOREM $3[5,78] .{ }^{2}$ Let $\lim _{n \rightarrow \infty} M_{n}^{1 / n}=\infty$. A necessary and sufficient condition that $C\left(M_{n}, k\right)$ be a quasi-analytic class is that

$$
\lim _{v \rightarrow \infty} H(v)=\infty,
$$

where $H(v)$ is defined by (2) and (3).

Definition 4. Let $\nu(x)$ be a function satisfying

(i) $\nu(x)$ is continuously differentiable for $0 \leqq x<\infty$,

(ii) $\nu(0)=1, \nu^{\prime}(x) \geqq 0,0 \leqq x<\infty$,

(iii) $x \nu^{\prime}(x) / \nu(x)=O(\log x)^{-1}, x \rightarrow \infty$.

We shall say that $f(x) \in C^{*}$ if and only if $f(x) \in C\left(M_{n}^{*}, k\right)$, where $M_{n}^{*}=n ![\nu(n)]^{n}$ and $\nu(n)$ satisfies the above conditions.

Definition 5. Let $Z(u)$ be a real-valued function which is less

Received by the editors November 29, 1954 and, in revised form, February 24, 1955.

${ }^{1}$ Presented to the Graduate School of Washington University in partial fulfillment of the requirements for the degree of Doctor of Philosophy. The basic idea used in the present proof is due to Professor I. I. Hirschman.

2 The first number in brackets refers to the references at the end of the paper; the remaining numbers, to the pages. 
than or equal to the number of zeros of $f(x)$, counted according to their multiplicities, in the closed interval $-u \leqq x \leqq u$.

We wish to give a new proof of the following theorem. It was first proved in a somewhat stronger form by Hirschman [2, 402].

Theorem 6. Let $f(x) \in C^{*}$, as in Definition 4. Let $C^{*}$ be a quasianalytic class. Let $H(v)$ be defined by (3). If there exists a function $Z(u)$ satisfying the conditions of Definition 5 such that

$$
\limsup _{u \rightarrow \infty} H[Z(u)] / u>k,
$$

then $f(x) \equiv 0$.

2. Results needed in the proof. The basic idea of the present proof makes use of the inversion theory of the class of convolution transforms with totally positive kernels, a theory which has been developed by Hirschman and Widder [3].

Let $\left(a_{n}\right)_{n-1}^{\infty}$ be a sequence of positive real numbers such that

$$
\sum_{n=1}^{\infty} a_{n}^{-2}<\infty, \quad \sum_{n=1}^{\infty} a_{n}^{-1}=\infty .
$$

Let $\left(b_{n}\right)_{n=0}^{\infty}$ be a sequence of real numbers satisfying

$$
\lim _{n \rightarrow \infty} b_{n}=0 \text {. }
$$

Let $s$ be the complex variable $s=\sigma+i \tau$, and define the entire functions

$$
E_{m}(s)=e^{b_{m} s} \prod_{n=m+1}^{\infty}\left(1-\frac{s}{a_{n}}\right) e^{s / a_{n}}, \quad m=0,1,2, \cdots
$$

The first of conditions (6) insures the convergence of the infinite product (8). The functions $G_{m}(t)$ are defined by

$$
G_{m}(t)=(2 \pi i)^{-1} \int_{-i \infty}^{i \infty} e^{s t}\left[E_{m}(s)\right]^{-1} d s, \quad m=0,1,2, \cdots .
$$

We shall write $E(s)=E_{0}(s), G(t)=G_{0}(t)$, and $b=b_{0}$. It can be shown $[3,144]$ that the integral $(9)$ converges absolutely for all real numbers t.

Theorem $7[3,143-144]$. If the functions $G_{m}(t)$ are defined by (9), $E_{m}(s)$ by (8), then

$$
\int_{-\infty}^{\infty} e^{-s t} G_{m}(t) d t=\left[E_{m}(s)\right]^{-1}, \quad m=0,1,2, \cdots,
$$


the integral converging absolutely for $-\infty<\sigma<\alpha$, where $\alpha=\min \left(a_{k},+\infty\right)$.

ThEOREM $8[3,155]$. If the functions $G_{m}(t)$ are defined by (9), then

(i) $G_{m}(t) \geqq 0,-\infty<t<\infty, m=0,1,2, \cdots$,

(ii) $\int_{-\infty}^{\infty} G_{m}(t) d t=1, m=0,1,2, \cdots$.

If we let $\phi(t)$ be a bounded and continuous function of $t$ on the whole real axis, it is clear from Theorem 8 that the convolution transform

$$
f(x)=\int_{-\infty}^{\infty} G(x-t) \phi(t) d t
$$

converges for all real values of $x$.

To solve the problem of inverting this transform, we introduce the linear differential operator

$$
P_{m}(D)=e^{\left(b-b_{m}\right) D} \prod_{n=1}^{m}\left(1-\frac{D}{a_{n}}\right) e^{D / a_{n}}
$$

where the operations $e^{a D}$ and $D$ applied to a function $f(x)$ mean that

$$
e^{a D} f(x)=f(x+a), \quad D f(x)=f^{\prime}(x) .
$$

It may be seen quite readily that

$$
P_{m}(D) G(t)=G_{m}(t), \quad-\infty<t<\infty .
$$

If we combine Theorem 8 (ii) with (12), we may show

Theorem $9[3,171,181,191]$. If $\phi(t)$ is a bounded continuous function of $t$, if $G(t)$ is the kernel function defined by (6), and if $f(x)$ is defined by the convolution transform (10), then

$$
\lim _{m \rightarrow \infty} P_{m}(D) f(x)=\phi(x), \quad-\infty<x<\infty .
$$

The proof of the following theorem depends on results due to Plancherel $[7,76]$ and to Kolmogoroff $[4,3]$. Only a sketch of the proof will be given here.

Theorem 10. Let $\psi(x)=\left(\pi^{-1 / 2} \sin x\right) / x, \omega(x)=\psi^{2}(x / 2)$. Define $F(x)=\omega(x) f(x)$. Let $\left(M_{n}\right)_{n=1}^{\infty}$ be a sequence of positive real numbers such that

(i) $\lim _{n \rightarrow \infty} M_{n}^{1 / n}=\infty$, and define $T(u)$ by (2). If

(ii) $f(x) \in C\left(M_{n}, k\right)$,

(iii) $\phi(t)=\int_{-\infty}^{\infty} e^{-i x t} F(x) d x$, 
and if $k_{1}$ is any number such that $k_{1}>k$, we have that

$$
\phi(t) T\left(|t| / k_{1}\right)=O(1), \quad \quad t \rightarrow \pm \infty .
$$

Proof. It is first necessary to show that, if $k_{1}>k$, then

$$
\int_{-\infty}^{\infty}|F(x)| d x \leqq A_{1} k_{1}^{n} M_{n}
$$

To show this, one uses the results mentioned above and proceeds in a manner similar to that in $[1,209]$.

Since $\omega^{(n)}(-\infty)=\omega^{(n)}(\infty)=0$ for $n=0,1,2, \cdots$, the same is true of $F^{(n)}( \pm \infty)$. Integrating by parts the transform which defines $\phi(t)$, we therefore obtain

$$
\phi(t)=\int_{-\infty}^{\infty} e^{-i t x}(i t)^{-n} F^{(n)}(x) d x .
$$

From (13) it follows that

$$
|\phi(t)| \leqq|t|^{-n} A_{1} k_{1}^{n} M_{n}, \quad n=0,1,2, \cdots .
$$

Applying the definition of $T(u)$, this yields the conclusion of our theorem,

$$
|\phi(t)| \leqq A_{1} / T\left(|t| / k_{1}\right) .
$$

By the symbol $f(x) \sim g(x)$, we mean that $\lim _{x \rightarrow \infty} f(x) / g(x)=1$.

Theorem 11 [2, 398-399]. Let $C^{*}$ be the class of functions of Definition 4. Let the function $\nu(x)$ satisfy (i), (ii), and (iii) of Definition 4. If $T(u)$ is defined by (2), then

$$
\log T(u) \sim u / \nu(u) .
$$

Lemma 12. Let $\nu(x)$ be the function defined in Definition 4. Then $\nu(a x) \sim \nu(x)$ for any $a>0$.

Proof. This is readily deduced from $x \nu^{\prime}(x) / \nu(x)=o(1)$ as $x \rightarrow \infty$.

Combining the results of Theorems 10,11 , and Lemma 12, we obtain

Theorem 13. Let $f(x) \in C^{*}$. Let

and define

$$
\omega(x)=\left[\frac{1}{\pi^{1 / 2}} \frac{\sin (x / 2)}{(x / 2)}\right]^{2},
$$

$$
F(x)=\omega(x) f(x), \quad \phi(t)=\frac{1}{(2 \pi)^{1 / 2}} \int_{-\infty}^{\infty} e^{-i x t} F(x) d x .
$$


For any $k_{1}>k$, we have that

$$
\phi(t)=O\left(\exp \left(\frac{-|t| q}{k_{1} \nu(|t|)}\right)\right)
$$$$
t \rightarrow \pm \infty
$$

3. Representation of quasi-analytic functions as convolution transforms.

LemmA 14. Let the class of functions $C^{*}$ be a quasi-analytic class. Let $\nu(x)$ be defined as in Definition 4. If we take $a_{n}=n \nu(n)$ for $n=1,2$, $3, \cdots$, then

$$
\sum_{n=1}^{\infty} a_{n}^{-2}<\infty, \quad \sum_{n=1}^{\infty} a_{n}^{-1}=\infty .
$$

Proof. The convergence of the first series follows from $\nu(n) \geqq 1$ and the comparison test. We conclude from Theorems 3 and 11 that

$$
\int_{1}^{\infty} \frac{d u}{u \nu(u)}
$$

diverges. Hence we have that $\sum_{n=1}^{\infty} a_{n}^{-1}=\infty$.

Theorem 15. Let $\nu(n)$ be defined as in Definition 4. Let $a_{n}=n \nu(n)$; $n=1,2,3, \cdots$. Let $E(s)$ be defined by

$$
E(s)=\prod_{n=1}^{\infty}\left(1-\frac{s}{a_{n}}\right) e^{s / a_{n}} .
$$

Then, if $\epsilon$ is an arbitrary positive number,

$$
E(i \tau)=O(\exp [(1+\epsilon)(\pi / 2)|\tau| / \nu(|\tau|)]), \quad \tau \rightarrow \pm \infty .
$$

Proof. We first define a function $n(u)$, which counts the zeros of $E(s)$. We set

$$
n(u)=n, n \nu(n) \leqq u \leqq(n+1) \nu(n+1), \quad n=0,1,2, \cdots .
$$

Since $\nu(n)$ is nondecreasing, we have that

$$
\frac{n \nu[n \nu(n)]}{(n+1) \nu(n+1)} \leqq \frac{n(u) \nu(u)}{u} \leqq \frac{\nu[(n+1) \nu(n+1)]}{\nu(n)},
$$

if $u$ lies in the interval $n \nu(n) \leqq u \leqq(n+1) \nu(n+1)$. We assert that

$$
\nu[n \nu(n)] \sim \nu(n) .
$$

For it follows from (iii) of Definition 4 that there exists a positive constant $M$ such that, if $n$ is sufficiently large, 


$$
\begin{aligned}
\log \frac{\nu[n \nu(n)]}{\nu(n)} & =\int_{n}^{n \nu(n)} \frac{\nu^{\prime}(x) d x}{\nu(x)} \leqq M \int_{n}^{n \nu(n)} \frac{d x}{x \log x} \\
& \leqq M \log \left[1+\frac{\log \nu(n)}{\log n}\right] .
\end{aligned}
$$

Thus we have that

$$
1 \leqq \frac{\nu[n \nu(n)]}{\nu(n)} \leqq\left[1+\frac{\log \nu(n)}{\log n}\right]^{M} .
$$

But it is also true that

$$
\log \frac{\nu(x)}{\nu(1)}=\int_{1}^{x} \frac{\nu^{\prime}(y) d y}{\nu(y)}=o(1) \log x, \quad x \rightarrow \infty,
$$

which implies that

$$
[\log \nu(n)] / \log n=o(1), \quad n \rightarrow \infty .
$$

Thus the right side of (19) tends to 1 as $n$ tends to infinity; hence, (18) is established. But now it follows from (17) that

$$
n(u) \sim u / v(u) .
$$

Let $\epsilon$ be an arbitrary positive number; there exists $u_{0}$ such that

$$
1-(2 \epsilon / \pi)<n(u) \nu(u) / u<1+(2 \epsilon / \pi), \quad u>u_{0} .
$$

Now we observe that

$$
\begin{aligned}
\log |E(i \tau)| & =\log \prod_{n=1}^{\infty}\left[1+\left(\tau^{2} / a_{k}^{2}\right)\right]^{1 / 2}=(1 / 2) \sum_{n=1}^{\infty} \log \left[1+\left(\tau^{2} / a_{k}^{2}\right)\right] \\
& =(1 / 2) \int_{0}^{\infty} \log \left[1+\left(\tau^{2} / u^{2}\right)\right] \operatorname{dn}(u) .
\end{aligned}
$$

An integration by parts yields

$$
\log |E(i \tau)|=\tau^{2} \int_{0}^{\infty}\left[n(u) / u\left(u^{2}+\tau^{2}\right)\right] d u .
$$

Thus it follows from (21) that

$$
\begin{aligned}
\log |E(i \tau)|< & \tau^{2} \int_{0}^{u_{0}} \frac{[n(u)-\{1+(2 \epsilon / \pi)\}\{u / \nu(u)\}] d u}{u\left(u^{2}+\tau^{2}\right)} \\
& +[1+(2 \epsilon / \pi)] \tau^{2} \int_{0}^{\infty} \frac{d u}{\nu(u)\left(u^{2}+\tau^{2}\right)}
\end{aligned}
$$




$$
\begin{aligned}
& \log |E(i \tau)|<O(1)+[1+(2 \epsilon / \pi)][|\tau| / \nu(|\tau|)] \\
& \cdot \int_{0}^{\infty} \frac{\nu(|\tau|)}{\nu(u)} \frac{|\tau| d u}{\left(u^{2}+\tau^{2}\right)}, \quad|\tau| \rightarrow \infty
\end{aligned}
$$

Setting

$$
I=\int_{0}^{\infty} \frac{\nu(|\tau|)}{\nu(u)} \cdot \frac{|\tau| d u}{\left(u^{2}+\tau^{2}\right)},
$$

we assert that $\lim _{|\tau| \rightarrow \infty} I=\int_{0}^{\infty}\left(1+v^{2}\right)^{-1} d v$. If we split the range of integration into the two intervals $[0,|\tau|]$ and $[|\tau|, \infty]$ and denote the resulting integrals by $I_{1}$ and $I_{2}$, respectively, we may take $u=v|\tau|$ to observe that

$$
I_{2}=\int_{1}^{\infty} \frac{\nu(|\tau|)}{\nu(v|\tau|)} \cdot \frac{d v}{\left(1+v^{2}\right)} .
$$

Since $v \geqq 1$, we have that $\nu(|\tau|) / \nu(v|\tau|) \leqq 1$; from Lemma 12, we also have that $\nu(v|\tau|) \sim \nu(|\tau|)$. Thus we may apply Lebesgue's limit theorem to conclude that

$$
\lim _{|\tau| \rightarrow \infty} I_{2}=\int_{1}^{\infty}\left(1+v^{2}\right)^{-1} d v
$$

Also, if $\delta$ is any real number such that $0<\delta<1$, there exists $u_{1}>0$ such that, for $b>a \geqq u_{1}$,

$$
\log [\nu(b) / \nu(a)]=\int_{a}^{b}\left[\nu^{\prime}(x) / \nu(x)\right] d x \leqq \delta \int_{a}^{b} d x / x=\delta \log (b / a),
$$

or

$$
[\nu(b) / \nu(a)] \leqq(b / a)^{8} .
$$

Taking $b=|\tau|, a=v|\tau|$, we conclude that

$$
[\nu(b) / \nu(a)] \leqq v^{-8}, \quad u_{1} /|\tau| \leqq v \leqq 1 .
$$

But now we may again set $u=v|\tau|$ to obtain

$$
\begin{array}{ll}
I_{1}=\int_{0}^{u_{1}} \frac{\nu(|\tau|)}{\nu(u)} \cdot \frac{|\tau| d u}{\left(u^{2}+\tau^{2}\right)}+\int_{u_{1} /|\tau|}^{1}[\nu(|\tau|) / \nu(v|\tau|)] \frac{d v}{\left(1+v^{2}\right)}, \\
I_{1}=o(1)+\int_{0}^{1} f_{\tau}(v)\left(1+v^{2}\right)^{-1} d v, & |\tau| \rightarrow \infty,
\end{array}
$$

where we have set 


$$
f_{\tau}(v)=\left\{\begin{array}{lr}
\nu(|\tau|) / \nu(v|\tau|), & u_{1} /|\tau| \leqq v \leqq 1 \\
0, & 0 \leqq v \leqq u_{1} /|\tau|
\end{array}\right.
$$

If we now define

$$
g(v)=\left\{\begin{array}{lr}
v^{-\delta} & 0<v \leqq 1, \\
0, & v=0,
\end{array}\right.
$$

it follows from the preceding remarks that

$$
f_{\tau}(v) \leqq g(v), \quad 0 \leqq v \leqq 1 .
$$

Also, if $0<v \leqq 1$, Lemma 12 states that $\nu(|\tau|) \sim \nu(v|\tau|)$. Thus

$$
\lim _{|\tau| \rightarrow \infty} f_{\tau}(v)=\left\{\begin{array}{lrl}
1, & 0<v \leqq 1, \\
0, & v & =0 .
\end{array}\right.
$$

Since $0<\delta<1, g(v) /\left(1+v^{2}\right)$ is an integrable function. Hence Lebesgue's limit theorem implies

$$
\lim _{|\tau| \rightarrow \infty} I_{1}=\int_{0}^{1}\left(1+v^{2}\right)^{-1} d v
$$

Letting $|\tau| \rightarrow \infty$, we obtain from (22) that

$$
\limsup _{|\tau| \rightarrow \infty} \frac{\log |E(i \tau)|}{|\tau| / \nu(|\tau|)} \leqq(\pi / 2)+\epsilon .
$$

In a similar fashion, we deduce from (21) that

$$
\liminf _{|\tau| \rightarrow \infty} \frac{\log |E(i \tau)|}{|\tau| / \nu(|\tau|)} \geqq(\pi / 2)-\epsilon .
$$

Since $\epsilon$ is arbitrary, we conclude that

$$
\log |E(i \tau)| \sim(\pi / 2)|\tau| / \nu(|\tau|) .
$$

The desired result (15) is an immediate consequence of (23).

Using the infinite product $E(s)$ defined in Theorem 15 , we introduce the kernel function

$$
G(t)=(2 \pi i)^{-1} \int_{-i \infty}^{i \infty} e^{s t}[E(s)]^{-1} d s .
$$

Theorem 16. Let $C^{*}$ be a quasi-analytic class, $f(x) \in C\left(M_{n}^{*}, k\right)$. Let $\omega(x)$ be defined as in Theorem 13. Set $h(x)=f(\alpha x)$ so that $h(x) \in C\left(M_{n}^{*}\right.$, $\alpha k)$. Set $\alpha=2 / \pi k_{1}$, where $k_{1}$ is any number such that $k_{1}>k$. Define

$$
F(x)=h(x) \omega(x) .
$$


There exists a bounded continuous function $\theta(t)$ such that

$$
F(x)=\int_{-\infty}^{\infty} G(x-t) \theta(t) d t,
$$

the convolution transform converging for all values of $x$.

Proof. We set

$$
\phi(t)=(2 \pi)^{-1 / 2} \int_{-\infty}^{\infty} e^{-i x t} F(x) d x
$$

Since $h(x) \in C\left(M_{n}^{*}, \alpha k\right)$, Theorem 13 implies that

$$
\phi(t)=O\left(\exp \left[-(\pi / 2) k^{\prime}|t| / \nu(|t|)\right]\right), \quad|t| \rightarrow \infty,
$$

where $k^{\prime}<k_{1} / k$. We choose $k^{\prime}>1$. Then we define the function $L(u)$ by

$$
L(u)=E(i u) \phi(u) .
$$

From (15) and (26), $L(u) \in L_{1}$. The boundedness and continuity of

$$
\theta(t)=(2 \pi)^{-1 / 2} \int_{-\infty}^{\infty} e^{i u t} L(u) d u
$$

are then simply elementary properties of Fourier transforms [7, 202].

Consider the integral

$$
\begin{aligned}
I & =\int_{-\infty}^{\infty} G(x-t) \theta(t) d t \\
& =(2 \pi)^{-1 / 2} \int_{-\infty}^{\infty} G(x-t) d t \int_{-\infty}^{\infty} e^{i u t} L(u) d u .
\end{aligned}
$$

By Theorem 8, and since $L(u) \in L_{1}$, this integral converges for every value of $x$ and may be inverted by Fubini's theorem to give

$$
I=(2 \pi)^{-1 / 2} \int_{-\infty}^{\infty} L(u) d u \int_{-\infty}^{\infty} G(x-t) e^{i u t} d t .
$$

Set $x-t=y$. Then by Theorem 7

$$
\int_{-\infty}^{\infty} G(x-t) e^{i u t} d t=\int_{-\infty}^{\infty} G(y) e^{i u(x-y)} d y=e^{i u x} / E(i u) .
$$

From (27) we see that

$$
I=(2 \pi)^{-1 / 2} \int_{-\infty}^{\infty} \phi(u) e^{i u x} d u .
$$


Since $\phi(u)$ and $F(x)$ both $\in L_{1}$, the inversion formula for the Fourier transform $[7,4]$ may be applied to deduce the desired relation (25)

$$
I=F(x)=\int_{-\infty}^{\infty} G(x-t) \theta(t) d t
$$

4. Proof of the main theorem. By the symbol $f(x) \leqq \sim g(x)$, we shall mean that $\lim \sup _{x \rightarrow \infty} f(x) / g(x) \leqq 1$. Let

Lemma 17 [2,398]. Let the class $C^{*}$ of Definition 4 be quasi-analytic.

$$
H(v)=(2 / \pi) \int_{1}^{v} u^{-2} \log T(u) d u
$$

Then

(i) $H(a x) \sim H(x), a>0, x \rightarrow \infty$.

(ii) $H\left(x_{1} x_{2}\right) \leqq \sim H\left(x_{1}\right)+H\left(x_{2}\right), x_{1}, x_{2} \rightarrow \infty$.

By Theorem 3, $H(v) \rightarrow \infty$ as $v \rightarrow \infty$. Thus the function $H^{*}(v)$ which is inverse to $H(v)$ is well defined for $0 \leqq v<\infty$ and

$$
v=(2 / \pi) \int_{1}^{H^{*}(v)} u^{-2} \log T(u) d u .
$$

We proceed to the proof of Theorem 6 .

Let $C^{*}$ be a quasi-analytic class, $f(x) \in C^{*}$. We assume that

$$
\limsup _{u \rightarrow \infty} H[Z(u)] / u=k^{\prime \prime}>k(=\infty),
$$

where $Z(u)$ is the function of Definition 5 . We wish to show that $f(x) \equiv 0$. Let $Z_{+}(u)$ be less than or equal to the number of zeros of $f(x)$ in the interval $0 \leqq x \leqq u$; let $Z_{-}(u)$ be less than or equal to the number of zeros of $f(x)$ in the interval $-u \leqq x \leqq 0$; require that $Z_{+}(u)$ $+Z_{-}(u)=Z(u)$. Then (29) implies that either or both of the relations

$$
\begin{aligned}
& \limsup _{u \rightarrow \infty} H\left[Z_{+}(u)\right] / u=k^{\prime \prime}, \\
& \limsup _{u \rightarrow \infty} H\left[Z_{-}(u)\right] / u=k^{\prime \prime},
\end{aligned}
$$

must be true. For we have that $H(2 x) \sim H(x)$ by Lemma 17 (i). Hence, if both of the limits superior (30) and (31) were less than $k^{\prime \prime}$, we would obtain a contradiction to (29). Thus we may assume that (30) is true.

Let $F(x)$ be defined as in Theorem 16. Let $W(u)$ be less than or 
equal to the number of zeros of $F(x)$ counted according to their multiplicities in the interval $0 \leqq x \leqq u$ and require that $W(u) \geqq Z_{+}(\alpha u)$. If we choose $k_{1}$ so that $k^{\prime \prime}>k_{1}>k$ and take $\alpha=2 / \pi k_{1}$ in Theorem 16 , it follows from (30) that

$$
\limsup _{u \rightarrow \infty} H[W(u)] / u \geqq \alpha k^{\prime \prime}>2 / \pi .
$$

But (32) implies that there exists a sequence $\left(N_{j}\right)_{j=1}^{\infty}, \lim _{j \rightarrow \infty} N_{j}=\infty$, and a real number $\epsilon>0$ for which

$$
W\left(N_{j}\right)>H^{*}\left[(2 / \pi)(1+\epsilon) N_{j}\right] .
$$

Divide the interval $\left[0, N_{j}\right]$ into $N_{j}^{2}$ subintervals of length $1 / N_{j}$. There will exist one subinterval $S_{j}$ which contains at least

$$
N_{i}^{-2} H^{*}\left[(2 / \pi)(1+\epsilon) N_{i}\right]
$$

zeros of the function $F(x)$. For if this were not the case, we would deduce a contradiction to (33).

Now we assert that, for any $a>0$,

$$
\lim _{v \rightarrow \infty} v^{-2} H(a v)=\infty .
$$

For, applying (28) and Theorem 11 , we have that

$$
v=(2 / \pi) \int_{1}^{H^{*}(v)} u^{-2} \log T(u) d u \sim(2 / \pi) \int_{1}^{H^{*}(v)} d u / u \nu(u) .
$$

For any real number $\epsilon>0$ and $v$ large enough, we deduce that

$$
(\pi / 2) v(1-\epsilon) \leqq \int_{1}^{H^{*}(v)} d u / u \nu(u) \leqq \int_{1}^{H^{*}(v)} d u / u=\log H^{*}(v),
$$

or

$$
\exp [(\pi / 2)(1-\epsilon) v] \leqq H^{*}(v),
$$

which implies (35). But from (35) we see that if the intervals $S_{j}$ remain bounded, then $f(x) \equiv 0$ follows from Definition 2 and Rolle's theorem.

Thus we may suppose that the intervals $S_{j}$ tend to infinity. Let $x$ be an arbitrary, but fixed, real number. We may suppose that $x$ lies to the left of the left-hand end point of each subinterval $S_{j}$; $j=1,2,3, \cdots$. Choose $n_{j}$ such that

$$
x+\sum_{k=1}^{n_{j}}[k \nu(k)]^{-1} \text { lies to the left of } S_{j},
$$




$$
x+\sum_{k=1}^{n_{j+1}}[k \nu(k)]^{-1} \text { lies in or to the right of } S_{j} .
$$

This is possible by Lemma 14 .

Recalling that each interval $S_{j}$ is contained in the interval $\left[0, N_{j}\right]$, we now estimate $n_{j}$. For any real number $\delta>0$ and $j$ sufficiently large, we have that

$$
\frac{\pi H\left(n_{j}\right)}{2(1+\delta)} \leqq \int_{1}^{n_{j}} \frac{d v}{v \nu(v)} \leqq \int_{1}^{n_{j}} \frac{1}{k \nu(k)} \leqq N_{j}-x,
$$

which yields

$$
n_{j} \leqq H^{*}\left[(2 / \pi)(1+\delta)\left(N_{j}-x\right)\right] .
$$

But now Lemma 17 (ii) may be used to establish that, if $v$ is sufficiently large, $\delta$ is sufficiently small, and $b$ is any constant,

$$
H^{*}[(2 / \pi)(1+\delta)(v+b)]<v^{-2} H^{*}[(2 / \pi)(1+\epsilon) v] .
$$

If we now observe that

$$
[1-(D / A)] f(x)=e^{A x} D e^{-A x} f(x) /(-A),
$$

we deduce from (34), (38), and (39) that there exists, by Rolle's theorem, at least one zero of the function

$$
\prod_{k=1}^{n_{i}}\left[1-\left(D / a_{k}\right)\right] F(x)
$$

in the interval $S_{j}$. Denote this zero by $\xi_{j}$. Choose $b=b_{0}=0$ and $\left(b_{j}\right)_{j=1}^{\infty}$ such that

$$
x+\sum_{k=1}^{n_{j}}[k \nu(k)]^{-1}+b_{j}=\xi_{j} .
$$

From (36) and (37) we see that

$$
\left|b_{j}\right|<\left(n_{j}+1\right)^{-1}+N_{j}^{-1} \text {. }
$$

Thus $\lim _{j \rightarrow \infty} b_{j}=0$.

Let $a_{k}=k \nu(k)$. Let $G(t)$ be the kernel function constructed in the proof of Theorem 16. That theorem asserts that there exists a continuous function $\theta(t)$ such that

$$
F(x)=\int_{-\infty}^{\infty} G(x-t) \theta(t) d t .
$$

If we take 


$$
P_{n_{j}}(D)=e^{b_{j} D} \prod_{k=1}^{n_{j}}\left[1-\left(D / a_{k}\right)\right] e^{D / a_{k}},
$$

it follows from Theorem 9 that

$$
\lim _{y \rightarrow \infty} P_{n_{j}}(D) F(x)=\theta(x) .
$$

But from (40) we have that

$$
P_{n_{j}}(D) F(x)=\prod_{k=1}^{n_{j}}\left[1-\left(D / a_{k}\right)\right] F\left(\xi_{j}\right)=0 .
$$

Hence we conclude that $\theta(x) \equiv 0$, since $x$ is arbitrary. But this means that $f(x) \equiv 0$.

\section{BIBLIOGRAPHY}

1. I. I. Hirschman, On the behaviour of Fourier transforms at infinity and on quasianalytic classes of functions, Amer. J. Math. vol. 72 (1950) pp. 200-213.

2. - On the distribution of the zeros of functions belonging to certain quasianalytic classes, Amer. J. Math. vol. 72 (1950) pp. 396-406.

3. I. I. Hirschman and D. V. Widder, The inversion of a general class of convolution transforms, Trans. Amer. Math. Soc. vol. 66 (1949) pp. 135-201.

4. A. Kolmogoroff, On inequalities between the upper bounds of the successive derivatives of an arbitrary function on an infinite interval, Amer. Math. Soc. Translation, no. 4, 1949.

5. S. Mandelbrojt, Analytic functions and classes of infinitely differentiable functions, The Rice Institute Pamphlet vol. 29 (1942) pp. 1-142.

6. E. C. Titchmarsh, The theory of functions, 2d ed., Oxford, 1939.

7. - Introduction to the theory of Fourier integrals, Oxford, 1948.

8. D. V. Widder and I. I. Hirschman, The convolution transform, Princeton, 1955.

WASHINGTON UNIVERSITY 